\title{
COGNITIVE RESERVE AND COGNITIVE PERFORMANCE IN HEALTHY LATVIAN SENIORS
}

\author{
Kristīne Šneidere \\ Riga Stradiṇš University, Latvia \\ Sonia Montemurro \\ University of Padua, Italy \\ Sara Mondini \\ University of Padua, Italy \\ Jelena Harlamova \\ Riga Stradiņš University, Latvia \\ Zane Ulmane \\ Riga Stradiņš University, Latvia \\ Ainārs Stepens \\ Riga Stradiņš University, Latvia
}

\begin{abstract}
In the next 30 years, a significant increase of the population aged over 65 is expected (WHO, 2015). Ageing can often be associated with cognitive decline; however, recent research indicates that symptoms of age-related cognitive impairment are modulated by Cognitive Reserve (CR), which derives from level of education, working activity, and social activity (Tucker \& Stern, 2011). The role of CR in neurodegenerative disease has been extensively researched, but little is known about its contribution to normal ageing. Fifty-four healthy Latvian seniors were recruited for the study. We assessed simple and complex reaction times, associative memory, memory retrieval, attention, working memory. Furthermore, we quantified their CR. We analysed the relationship between $C R$ and reaction times with two separate logistic regressions. Then, four linear regression models were built to analyse the relationship between $C R$ and the scores on the cognitive tasks. CR was not related to the cognitive performance of healthy Latvian seniors. Such results indicate that CR resources may be mostly required when high-demanding tasks have to be performed.
\end{abstract}

Keywords: ageing, cognitive performance, cognitive reserve.

\section{Introduction}

Ageing has become one of the core issues regarding health and wellbeing, with the World Health Organization (WHO) predicting in next 30 years a significantly increased number of people aged over 65 (WHO, 2015). Ageing produces changes not only in the biological domain, but also in psychological and 
social contexts. Such changes sometimes generate a decrease in brain volume (Bherer, Erickson, \& Liu-Ambrose, 2013; Colcombe et al., 2003), or declined cognitive functioning (Morra, Zade, McGlinchey, \& Milberg, 2016). Previous studies indicate that the most vulnerable cognitive functions in ageing are the executive functions (e.g., inhibition, working memory, cognitive flexibility, updating and shifting) (Boucard et al., 2012; Diamond, 2013), memory encoding, episodic memory (Hedden \& Gabrieli, 2004; Reuter-Lorenz \& Park, 2010; Salthouse, 2010), and processing speed (Baudouin, Clarys, Vanneste, \& Isingrini, 2009).

The ability to overcome neuropathology in ageing by using previously developed cognitive or compensational processes can be modulated by Cognitive reserve (CR) (Stern, 2009). Educational level, working activity, free time and social activities, IQ (Stern, 2009; Tucker \& Stern, 2011) and bilingualism (Guzmán-Vélez \& Tranel, 2015) are considered proxies of CR. Yaakov Stern has indicated two main mechanisms underlying CR, that is neural reserve and neural compensation. Neural reserve refers to increased efficiency of existing functional neural resources and the ability to overcome the impact of brain damage or pathological cognitive decline (e.g., mild cognitive impairment), while neural compensation can be considered as the ability to recruit new, or additional, functional resources. Neural compensation is associated with the ability to use different neural pathways, either in the presence of neuropathology or while conducting high-demanding cognitive tasks (Steffener, Reuben, Rakitin, \& Stern, 2011; Stern, 2009; Tucker \& Stern, 2011).

A number of studies have focused on the contribution of CR to mild cognitive impairment (MCI) and Alzheimer's disease (AD). However, research on the relationship between CR and cognitive decline in normal ageing has only recently started and results have been contradictory. A recent meta-analysis conducted by Opdebeeck, Martyr and Clare (2016) has indicated that in healthy seniors there is an association between CR and cognitive performance in memory, executive functions, working memory, visuospatial abilities, language tasks, and with level of IQ. However, there is very little evidence on the relationship between CR and "fluid" cognitive functions (Baltes, 1999), such as processing speed and reaction times (Lavrencic, Churches, \& Keage, 2017; Ritchie, Bates, Der, Starr, \& Deary, 2013), episodic memory, working memory or executive functions (Thow et al., 2018).

The aim of this study is to examine whether level of CR predicts performance of working memory, associative memory, attention and processing speed. 


\section{Materials and methods}

\section{Participants}

To assess the relevance of cognitive reserve on cognitive functioning, fiftyfour healthy Latvian seniors aged from 65 to 85 ( $M=71.44, S D=4.91,25 \%$ male) were recruited. We adopted inclusion/exclusion criteria on the basis of medical history. Thus, we excluded participants with cardio-vascular diseases, metabolic disorders, pulmonary and respiratory diseases requiring regular inhalator use, ongoing oncological diseases, rheumatic diseases requiring regular medication intake, and mental diseases. Data were obtained during a two-year time span.

\section{Measures}

We acquired demographic data through the Demographic data questionnaire, which consists of different questions regarding health and health behaviour of participants, as well as questions regarding their socio-economic status.

The Montreal Cognitive Assessment (MoCA, Nasreddine et al., 2005) was used as a measure of global cognitive score. The MoCA test is an instrument to assess mild cognitive dysfunction and it evaluates different cognitive domains.

We assessed long-term memory retrieval and associative memory with the subtest Memory for Names from the Woodcock-Johnson Test of Cognitive Abilities (Woodcock, McGrew, \& Mather, 2001). Participants were presented with pictures of cartoon-like aliens with their names and then asked to recognise both the picture and the name of each alien.

Working memory was assessed with Numbers Reversed test, another subtest from Woodcock-Johnson Test of Cognitive Abilities. Participants had to repeat a series of numbers in reverse order (Woodcock, McGrew, \& Mather, 2001).

The Map Test of Everyday Attention (Robertson, Ward, Ridgeway, \& Nimmo-Smith, 1996) was used to measure attention, concentration and information processing speed. Participants were asked to mark on a map as many pictures of a gas station as possible.

Attention and concentration were also assessed with Visual Matching test from Woodcock-Johnson Test of Cognitive Abilities (Woodcock, McGrew, \& Mather, 2001). Participants were presented with a set of rows of 5 numbers, where two of the numbers were identical. They were asked to mark the two identical numbers present in each row. In all Woodcock-Johnson subtests, standardized score was used.

Simple and choice reaction times were measured by two computerized reaction time tasks, that is: (1) simple reaction times task (SRT; participants had to react to a visual stimulus by pressing a button on the keyboard as fast as they could) and (2) choice reaction times task (CRT; participants were asked to detect 
the left-or-right position of the same visual stimulus and press the corresponding button on the keyboard; Molotanovs, 2013). A training phase preceded each assessment.

To measure participants' cognitive reserve we used the Cognitive Reserve Index questionnaire (CRIq; Nucci, Mapelli, \& Mondini, 2012, adapted in Latvian by Šneidere \& Harlamova, 2017). The questionnaire is used for a standardized estimation of the cognitive reserve accumulated by individuals through their life, including demographic data and three sections: education, working activity, and leisure time, each of which returned a sub-score.

\section{Procedure}

Participants were recruited via retirement associations and mass media. Data were obtained separately from each participant. Before being asked to take part in the study, the healthy seniors were interviewed by telephone to determine their compliance with the inclusion/exclusion criteria. All participants were righthanded, with good or corrected vision and hearing. Before the assessment, the aims of the study were explained, and the ethical aspects elucidated. Participation was voluntary. Ethical approval was obtained from the Riga Stradiňš University Ethics Committee.

\section{Data analysis}

A null model (i.e., Model 0) was built for each dependent variable respectively. All models were built by entering the CRI total score in the null model, then adjusting the test scores for the effect of CRI in a series of regression analyses. In two separate logistic regressions, the mean value of Simple Reaction Time (SRT; Model 1) and Choice Reaction Time (CRT; Model 2) were considered as dependent variables. Then, four linear regression models were separately built with the CRI total score as predictor and the Map Test of Everyday Attention (Model 3), the Memory for Names (Model 4), the Visual Matching test (Model 5) and the Numbers Reversed Test (Model 6) as dependent variables.

The global cognitive performance of participants (i.e., MoCA score) was entered as dependent variable and the CRI score as independent variable in Model 7. Outliers' influence was checked by means of the Cook's distance (Di) (Cook \& Weisberg, 1982). Data distribution of each dependent variable was checked before the analyses. The Bayesian Information Criterion (BIC) was used for checking consistency of the models (Neath \& Cavanaugh, 2012). The difference between consistency of Model 0 and consistency of models with CRI as predictor, was calculated by means of delta-BIC $(\triangle \mathrm{BIC})$, which explains "how many times" the Model 0 fits the data better than the models with CRI as predictor. Delta-BIC was calculated on each dependent variable. A series of correlations was performed to measure the association between CRI sub-components (i.e. CRI-Education, CRI-WorkingActivity and CRI-LeisureTime; Nucci et al., 2012) 
and the dependent variables (see Table 1). All the analyses were performed by using R Software (R Core Team. Version 3.3.1, 2017) and an alpha level of 0.05 was set to determine significance.

\section{Results}

One observation was deleted due to missing values; one observation was deleted due to the presence of influential outliers ( $\mathrm{Di}>1$; Cook \& Weisberg, 1982).

\section{Table 1 Correlation matrix}

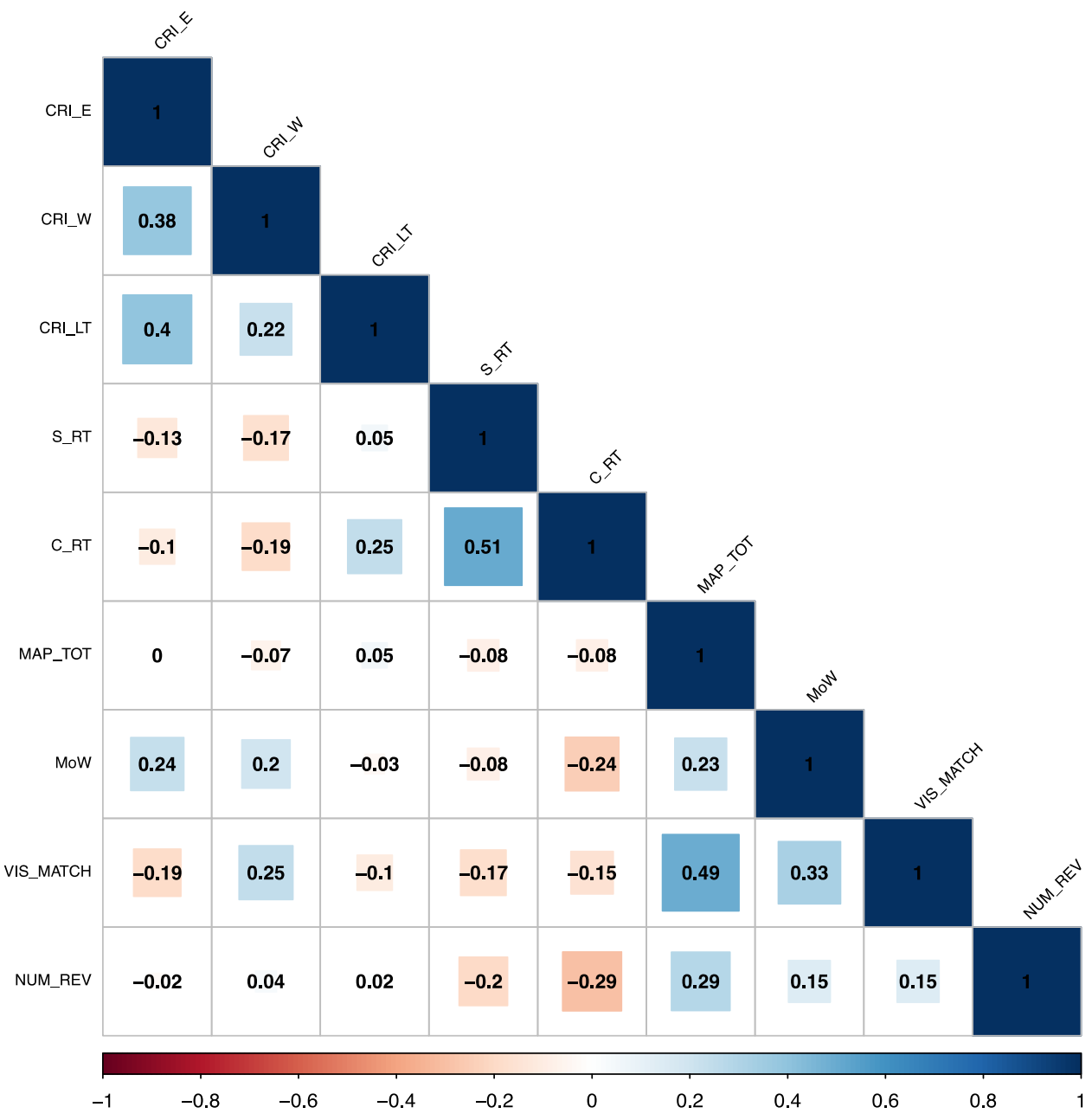

Notes: Explanation of acronyms: CRI_E - Cognitive Reserve Subindex "Education”, CRI_W Cognitive Reserve Subindex "Working Activity", CRI_LT - Cognitive Reserve Subindex "Leisure Time”, S_RT - simple reaction time, C_RT - choice reaction time, MAP_TOT - The Map test total score, MoW - Memory of Names task, VS_MATCH - Visual Matching test, NUM_REV - the Numbers Reversed test. 
In Model 1 and Model 2 (i.e., Simple and Choice RT), adding the CRI as predictor did not produce significant improvement in the fit of the model $\left[\mathrm{t}_{(52)}=\right.$ $\left.0.81, p=0.41 ; \mathrm{t}_{(52)}=0.23, p=0.78\right]$. In the same way, CRI did not improve the model fit of any cognitive score, compared to their baseline (i.e., Model 0): Map Test (Model 3: $\mathrm{R}^{2}=0.0003, p=0.89$ ), Memory of Names (Model 4: $\mathrm{R}^{2}=0.032$, $p=0.19$ ), Visual Matching (Model 5: $\mathrm{R}^{2}=0.001, p=0.78$ ), and Numbers Reversed Test (Model 6: $\mathrm{R}^{2}=0.0002, p=0.9$ ). The Global cognitive score (MoCA) did not show any relationship with the CRI total score (Model 7: $\mathrm{R}^{2}=$ $0.003, p=0.65$ ).

Each Model 0 (i.e., the model with no predictors for each dependent variable) generally predicted our data distribution better than the models with CRI as predictor; that is, in the Simple Reaction Times task, Model 0 was 3.9 times better than Model 1. For the Choice Reaction Time task, Model 0 was 7.03 times better than Model 2. Similar results were found for the Map Test $(\triangle \mathrm{BIC}=7.28)$, the Memory of Names test $(\triangle \mathrm{BIC}=3.02)$, the Visual Matching test $(\triangle \mathrm{BIC}=7.07)$, the Numbers Reversed test $(\triangle \mathrm{BIC}=7.29)$ and the MoCA test $(\triangle \mathrm{BIC}=6.61)$. Participants with high CRI-Education and CRI-Working Activity were faster in the Complex Reaction Times task (see Table 1). Correlations of CRI sub-scores with participants' performance showed a very heterogeneous pattern. Only in the performance on the Numbers Reversed test, participants showed relevant low relationship with all CRI sub-components.

\section{Discussion}

The aim of our study was to examine whether the level of cognitive reserve (CR) can predict performance in working memory, associative memory, attention and processing speed tasks in healthy Latvian older persons. Our main results indicate that CR is not significantly associated with simple reaction times, or with choice reaction times. Similarly, CR was not associated with performance in working memory, associative memory and memory retrieval and attention tasks. Our results might suggest that CR does not modulate specific cognitive performances in this group of healthy seniors. This could be due to the complexity of the tasks. Previous studies (which investigated the relationship between CR and neural reserve) have found that as the complexity of verbal working memory tasks increased participants with high CR presented reduced neural activity and faster time responses. This suggests greater neural efficiency with high CR especially in the elderly (Speer \& Soldan, 2015). Similarly, in a study by Gu et al. (2018) in which the effect of CR on brain activation in MCI patients and healthy seniors was investigated, healthy seniors with higher CR showed lower task-related activity. 
The above-mentioned studies confirm the results found by Ansado et al. (2013), who examined both neural compensation and neural reserve in attention and in speediness tasks. Participants were divided into two age groups (i.e., younger adults and older adults). In high-demanding tasks, older adults had similar results to the younger participants. However, in a low-demanding task the younger group was significantly faster than the older group.

While objective measures in pathological ageing indicate the beneficial role of CR, the relationship between cognitive performance and CR in healthy people is less clear. A meta-analysis conducted by Opdebeeck and colleagues (Opdebeeck et al., 2016) indicated that there are specific cognitive processes that might be related either to a composite score for CR, or to specific CR proxies (e.g., education, working activity or social activity). Furthermore, it has been found that $\mathrm{CR}$ is associated with working memory, verbal memory, executive functions, verbal fluency and verbal interference tasks (Lavrencic, Churches, \& Keage, 2017), but no relationship has been shown between processing speed and CR. Such results only partially support our findings. This can be due to the use of proxies of CR (e.g., education) instead of specific measures of CR components. Instead, we adopted a composite measure of CR (CRIq; Nucci et al., 2012).

The present study has some limitations. First, the sample was rather small; second, an objective measure (e.g., measurement of event-related potentials during cognitive performance) was not included. In the future, research should be longitudinal in order to measure not only cognitive performance, but also its decline.

\section{Conclusion}

The present study indicates that CR might not be beneficial in tasks measuring simple and choice reaction times, working memory, associative memory and attention in healthy seniors. While previous research confirms a relationship between CR and the later onset of symptoms of neurodegeneration, we suggest that in healthy ageing CR might be beneficial only in very highdemanding tasks.

As already said, the applicability of the data is limited by the sample size and lack of objective measures. To adequately measure the relationship between CR and normal age-related decline in cognitive functioning, a longitudinal research would be beneficial.

\section{Acknowledgements}

This study was conducted under the State Research Programme BIOMEDICINE, subproject No. 5.8.2. 
Šneidere et al., 2018. Cognitive Reserve and Cognitive Performance in Healthy Latvian Seniors

\section{References}

Ansado, J., Monchi, O., Ennabil, N., Deslauriers, J., Jubault, T., Faure, S., \& Joanette, Y. (2013). Coping with task demand in aging using neural compensation and neural reserve triggers primarily intra-hemispheric-based neurofunctional reorganization. Neuroscience research, 75 (4), 295-304.

Baltes, P. B., Staudinger, U. M., \& Lindenberger, U. (1999). Lifespan psychology: Theory and application to intellectual functioning. Annual review of psychology, 50 (1), 471-507.

Baudouin, A., Clarys, D., Vanneste, S., \& Isingrini, M. (2009). Executive functioning and processing speed in age-related differences in memory: Contribution of a coding task. Brain and Cognition, 71 (3), 240-245. https://doi.org/10.1016/j.bandc.2009.08.007

Bherer, L., Erickson, K. I., \& Liu-ambrose, T. (2013). A Review of the Effects of Physical Activity and Exercise on Cognitive and Brain Functions in Older Adults, 2013.

Boucard, G. K., Albinet, C. T., Bugaiska, A., Bouquet, C. A., Clarys, D., \& Audiffren, M. (2012). Impact of Physical Activity on Executive Functions in Aging: A Selective Effect on Inhibition Among Old Adults, Journal of Sport and Exercise Psychology, 34 (6), 808827.

Colcombe, S. J., Erickson, K. I., Raz, N., Webb, A. G., Cohen, N. J., McAuley, E., \& Kramer, A. F. (2003). Aerobic fitness reduces brain tissue loss in aging humans. The Journals of Gerontology Series A: Biological Sciences and Medical Sciences, 58 (2), M176-M180.

Cook, R. D., \& Weisberg, S. (1982). Residuals and influence in regression. New York: Chapman and Hall.

Darby, R. R., Brickhouse, M., Wolk, D. A., \& Dickerson, B. C. (2017). Effects of cognitive reserve depend on executive and semantic demands of the task. Journal of Neurology, Neurosurgery \& Psychiatry, jnnp-2017-315719. https://doi.org/10.1136/jnnp-2017315719

Diamond, A. (2013). Executive Functions. Annual Review of Psychology, 64 (1), 135-168. https://doi.org/10.1146/annurev-psych-113011-143750

Gu, L., Chen, J., Gao, L., Shu, H., Wang, Z., Liu, D., ... \& Zhang, Z. (2018). Cognitive reserve modulates attention processes in healthy elderly and amnestic mild cognitive impairment: An event-related potential study. Clinical Neurophysiology, 129 (1), 198-207.

Guzmán-Vélez, E., \& Tranel, D. (2015). Does bilingualism contribute to cognitive reserve? Cognitive and neural perspectives. Neuropsychology, 29 1), 139-150. https://doi.org/10.1037/neu0000105

Hedden, T., \& Gabrieli, J. D. E. (2004). Insights into the ageing mind: a view from cognitive neuroscience. Nature Reviews Neuroscience, 5 (2), 87-96. https://doi.org/10.1038/ nrn1323

Lavrencic, L. M., Churches, O. F., \& Keage, H. A. (2017). Cognitive reserve is not associated with improved performance in all cognitive domains. Applied Neuropsychology: Adult, $1-13$.

Molotanovs, A. (2013). Sacensību darbības optimizēšana handbola vārtsargiem (uz HK LSPA komandas piemēra). Latvian Academy of Sports Pedagogy. Retrieved from http://lspa.eu/files/students/Promotion/Andris_MOLOTANOVS_promocijas_darbs.pdf

Morra, L., Zade, D., McGlinchey, R. E., \& Milberg, W. P. (2013). Normal aging and cognition: The unacknowledged contribution of cerebrovascular risk factors. Aging, Neuropsychology, and Cognition, 20 (3), 271-297.

Nasreddine, Z. S., Phillips, N. A., Bédirian, V., Charbonneau, S., Whitehead, V., Collin, I., ... 
\& Chertkow, H. (2005). The Montreal Cognitive Assessment, MoCA: a brief screening tool for mild cognitive impairment. Journal of the American Geriatrics Society, 53 (4), 695-699.

Nucci, M., Mapelli, D., \& Mondini, S. (2012). Cognitive Reserve Index questionnaire (CRIq): a new instrument for measuring cognitive reserve. Aging Clinical and Experimental Research, 24 (3), 218-226.

Opdebeeck, C., Martyr, A., \& Clare, L. (2016). Cognitive reserve and cognitive function in healthy older people: A meta-analysis. Aging, Neuropsychology, and Cognition, 23( 1), 40-60. https://doi.org/10.1080/13825585.2015.1041450

Reuter-Lorenz, P. A., \& Park, D. C. (2010). Human neuroscience and the aging mind: a new look at old problems. The Journals of Gerontology. Series B, Psychological Sciences and Social Sciences, 65 (4), 405-15. https://doi.org/10.1093/geronb/gbq035

Ritchie, S. J., Bates, T. C., Der, G., Starr, J. M., \& Deary, I. J. (2013). Education is associated with higher later life IQ scores, but not with faster cognitive processing speed. Psychology and Aging, 28 (2), 515-521. https://doi.org/10.1037/a0030820

Robertson, I. H., Ward, T., Ridgeway, V., \& Nimmo-Smith, I. (1996). The structure of normal human attention: The Test of Everyday Attention. Journal of the International Neuropsychological Society, 2 (6), 525-534.

Salthouse, T. A. (2010). Selective review of cognitive aging. Journal of the International neuropsychological Society, 16 (5), 754-760. https://doi.org/10.1017/S135561771000 0706

Sneidere, K., \& Harlamova, J. (2017). Impact of aerobic activities on cognitive functioning Unpublished Master's Thesis, Riga Stradiňš University, Riga, Latvia.

Speer, M. E., \& Soldan, A. (2015). Neurobiology of Aging Cognitive reserve modulates ERPs associated with verbal working memory in healthy younger and older adults. Neurobiology of Aging, 36 (3), 1424-1434. https://doi.org/10.1016/j.neurobiolaging. 2014.12.025

Steffener, J., Reuben, A., Rakitin, B. C., \& Stern, Y. (2011). Supporting performance in the face of age-related neural changes: testing mechanistic roles of cognitive reserve. Brain Imaging and Behavior, 5 (3), 212-221. https://doi.org/10.1007/s11682-011-9125-4

Stern, Y. (2009). Cognitive reserve. Neuropsychologia, 47 10), 2015-28. https://doi.org/ 10.1016/j.neuropsychologia.2009.03.004

Thow, M. E., Summers, M. J., Saunders, N. L., Summers, J. J., Ritchie, K., \& Vickers, J. C. (2018). Further education improves cognitive reserve and triggers improvement in selective cognitive functions in older adults: The Tasmanian Healthy Brain Project. Alzheimer's and Dementia: Diagnosis, Assessment and Disease Monitoring, 10, 22-30. https://doi.org/10.1016/j.dadm.2017.08.004

Tucker, A., \& Stern, Y. (2011). Cognitive reserve in aging. Current Alzheimer Research, 8 (4), 354-360.

Woodcock, R. W., McGrew, K. S., \& Mather, N. (2001). Woodcock-Johnson III Test Manual (III). Itasca, IL: Riverside Publishing Company.

World Health Organization. (2015). World report on Ageing And Health. Retrieved from www.who.int/ 